FORMATION Formation emploi

Revue française de sciences sociales

148 | Octobre-Décembre

Varia

\title{
Le jeu international des business schools chinoises : entre excellence académique et pertinence professionnelle
}

Chinese business schools international game. Between academic excellence and professional relevance.

Das internationale Match der chinesischen Business Schools : zwischen

akademischer Exzellenz und professioneller Relevanz

El juego internacional de las escuelas de negocios chinas : entre excelencia académica y pertinencia profesional

\section{Tupac Soulas}

\section{OpenEdition}

Journals

Édition électronique

URL : http://journals.openedition.org/formationemploi/7877

DOI : 10.4000/formationemploi.7877

ISSN : 2107-0946

Éditeur

La Documentation française

Édition imprimée

Date de publication : 31 décembre 2019

Pagination : 87-106

ISSN : 0759-6340

Référence électronique

Tupac Soulas, «Le jeu international des business schools chinoises : entre excellence académique et pertinence professionnelle », Formation emploi [En ligne], 148 | Octobre-Décembre, mis en ligne le 04 janvier 2021, consulté le 07 janvier 2021. URL : http://journals.openedition.org/formationemploi/7877 ; DOI : https://doi.org/10.4000/formationemploi.7877 


\title{
Le jeu international des business schools chinoises : entre excellence académique et pertinence professionnelle
}

\author{
TUPAC SOULAS \\ Sinologue et docteur en sociologie, spécialiste des relations internationales des organisations \\ d'enseignement supérieur et de la Chine. Chercheur indépendant
}

Résumé

\begin{abstract}
Le jeu international des business schools chinoises : entre excellence académique et pertinence professionnelle
\end{abstract}

Cette étude analyse la transformation d'écoles de gestion chinoises prestigieuses qui intègrent le champ international des écoles de gestion. Pour cela, leur corps professoral doit exceller à la fois dans son orientation académique et professionnelle. La solution adoptée est la scission du corps professoral entre spécialistes du monde professionnel et du monde académique. Ces changements visent à rendre la composition du corps professoral plus lisible dans le champ international et sont largement inspirés des préconisations des agences d'accréditations et instances de classement internationales.

Mots clés : enseignement supérieur, diplôme de gestion, établissement d'enseignement, évaluation, projet d'établissement, enseignant, pratique de GRH, Chine

Abstract

Chinese business schools international game. Between academic excellence and professional relevance.

The study analyses Chinese schools of management transformation in order to enter the international field of schools of management. For that purpose, their faculty must excel in both academic and professional orientation. The favoured solution is the division of the faculty between scholars and practioners. These changes intend to make the composition of the faculty readable in the international field and are mostly inspired by accreditation agencies and ranking organizations.

Keywords: higher education, management degree, educational institution, evaluation, school work plan, teacher, HR management practice, China

Journal of Economic Literature: I 23 ; M 54

Traduction : auteur. 


\section{Introduction}

Les écoles de gestion d'Europe ou d'Amérique du Nord sont des organisations évoluant dans un monde que leurs responsables qualifient de très compétitif. Les doyens des "business schools" sont choisis pour piloter ces écoles dans un contexte d'évaluation permanente, rythmée par les accréditations et classements internationaux. La réactivité des écoles les plus prestigieuses est aujourd'hui si forte qu'elles entreprennent des transformations radicales. Cet article analyse l'un de ces changements en montrant que le monde des "business schools " produit des normes à l'échelle internationale qui conduisent les écoles à scinder leur corps professoral.

Nous définirons une école de gestion comme un établissement d'enseignement supérieur dispensant des cours en gestion au sein d'une université (faculté de gestion) ou de manière indépendante (comme les écoles de commerce françaises). La gestion est un domaine comprenant les disciplines relatives à l'administration des entreprises, dans un contexte d'économie de marché (finance, marketing, comptabilité, commerce, etc.) et qui fut enseignée, en Chine, dès le début du $20^{\text {ème }}$ siècle. Par simplicité, nous désignerons par "Chine » le territoire actuel de la République Populaire de Chine (RPC), qui inclut la Chine continentale, mais également les régions de Hong Kong et Macao.

\section{Le champ international des écoles de gestion}

En Amérique du Nord, en Europe et en Chine notamment, les écoles de gestion cherchent à se positionner dans un champ d'action stratégique : le champ international des écoles de gestion (Soulas, 2016, pp. 35-67). Nous nous appuyons sur le travail de Fligstein \& McAdam (2012, p. 9) qui définissent un champ d'action stratégique comme un ordre social construit de niveau intermédiaire (meso), dans lequel des acteurs interagissent et s'adaptent les uns aux autres sur la base d'une vision partagée des objectifs du champ, des relations avec les autres dans le champ et des règles qui y gouvernent l'action légitime.

Partant de cette définition, nous considérerons qu'il existe un champ international d'action stratégique des écoles de gestion créé par l'exportation de pratiques développées en Amérique du Nord. Il véhicule un modèle d'école de gestion inspiré des établissements états-uniens qui ont un rôle dominant dans ce champ.

Ce que nous désignons par modèle de la business school est constitué de trois prescriptions : l'offre de programmes professionnels (dont le fameux Master of Business Administration ou MBA), une internationalisation forte de toutes les dimensions de l'école (programmes, corps étudiant et professoral, etc.), et l'investissement de ressources dans les activités de recherche (notamment la publication dans des revues nordaméricaines). Ces normes sont portées et sanctionnées par les unités de gouvernance interne de ce champ, à savoir les trois principales agences d'accréditations internatio- 
nales (AACSB, EFMD et $\mathrm{AMBA}^{1}$ ) et le principal classement mondial, celui du magazine Financial Times, qui domine aujourd'hui l'évaluation des écoles à cette échelle.

En résumé, un grand nombre des écoles dans le monde cherchent à bénéficier des ressources et de la légitimité que confère l'appartenance au champ international. Pour cela, elles doivent correspondre au modèle de la business school par ces trois prescriptions dont l'adoption est évaluée et validée par les unités de gouvernance interne du champ : les agences d'accréditations et instances de classement sus-nommées.

\section{Des écoles aux orientations académiques et professionnelles}

L'intégration de normes internationales dans les organisations d'enseignement supérieur est souvent perçue à travers l'affirmation de règles emblématiques, comme celle du publish or perish ${ }^{2}$ dans les revues états-uniennes.

Les écoles de gestion ont une spécificité : elles s'appuient sur deux sources de légitimité, académique et professionnelle. La légitimité académique est fondée sur les normes d'évaluation du monde universitaire auquel les écoles sont rattachées. Elle les conduit à s'affirmer comme des acteurs de production scientifique : ouverture de centres de recherche ; formations doctorales ; recrutement de chercheurs, etc. La légitimité professionnelle repose, quant à elle, sur la pertinence de leurs activités vis-à-vis du monde des affaires : placement des étudiants sur le marché du travail ; collaborations avec les entreprises ; enseignants délivrant des formations aux professionnels, etc.

Ces deux orientations conduisent les écoles à être reconnues à la fois par les universités et par les cabinets de conseil en entreprise. La recherche de ces deux sources de légitimité a évolué dans le temps, et ces orientations n’ont pas un poids identique dans toutes les écoles. Certaines écoles sont largement marquées par leur insertion dans le monde académique. La plupart des écoles universitaires états-uniennes s'y inscrivent très largement. D'autres écoles ont une forte autonomie à l'égard des structures universitaires de leur pays, comme les grandes écoles françaises jusque dans les années 1970 (Blanchard 2012, p. 419). Aux États-Unis, en Europe, mais également en Chine, ces deux sources de légitimité coexistent, les écoles opérant des choix d'équilibre quant à l'importance accordée à chacune.

1. L'Association to Advance Collegiate Schools of Business (AACSB) est une association d'établissements basée aux États-Unis. L'European Foundation for Management Development (EFMD) est une association d'établissements d'origine européenne, tandis que l'Association of $M B A s$ (AMBA) est d'origine britannique.

2. publier ou périr. 


\section{Comment le champ international transforme les écoles}

Aujourd'hui, les écoles de gestion les plus prestigieuses doivent faire preuve d'excellence dans le domaine académique, tout en demeurant pertinentes pour les acteurs du monde des affaires. Cela implique d'avoir des professeurs à la fois capables des productions scientifiques les plus avancées de leur discipline et possédant des compétences techniques et pédagogiques permettant d'enseigner la pratique des affaires et de conseiller les entreprises dans leur gestion. Ce constat est fait par les membres des écoles de gestion les plus prestigieuses et critiqué par beaucoup de professeurs, mais nous ne chercherons pas à juger du bien-fondé de cette double exigence.

Dans cet article, nous allons analyser comment sont construites ces préconisations envers les écoles de gestion du champ international et montrer comment ces dernières en viennent à élaborer la division de leur corps professoral. Nous mettrons ainsi en évidence l'importance de la place de chaque école dans le champ international pour expliquer les réponses apportées par chacune à cette double injonction. Ainsi, les écoles les mieux insérées dans le champ reprennent presque à la lettre les règles émises par ces unités de gouvernance interne, alors que les nouveaux arrivants adoptent des solutions qui répondent en priorité aux règles de leur champ local.

\section{Une analyse basée sur une enquête de terrain approfondie}

L'analyse s'appuie sur un travail de terrain mené durant un total de douze mois d'enquête, entre 2010 et 2014, au sein de trois écoles de gestion prestigieuses de grandes villes chinoises : l'Institut Nanzhu à Canton, HKNU Business School à Hong Kong et Asia Business School (ABS) à Shanghai ${ }^{3}$. Elles appartiennent aux trois champs locaux identifiés en Chine (respectivement les systèmes universitaires de Chine continentale et de Hong Kong, et les écoles hors système éducatif ${ }^{4}$ ).

Des observations ont été menées dans les trois établissements, mais les données sont essentiellement constituées de 116 entretiens semi-directifs avec des membres des écoles, mais aussi des membres de leur université ou d'autres établissements du champ local. Les enquêtés ont été choisis selon les contacts obtenus, mais en recherchant un équilibre des catégories d'acteurs au sein de l'école (professeurs, étudiants, personnels) et en respectant aussi les divisions internes de ces groupes ( $c f$. Tableau 1).

3. Les noms des personnes et des institutions mentionnées dans cet article sont anonymisés.

4. Ces trois champs locaux des écoles chinoises sont décrits dans la partie 2. 
TABLEAU 1 - Répartitions des entretiens dans les trois terrains

\begin{tabular}{lccc}
\hline Enquêtés & HKNU Business School & Asia Bunisess School & Institut Nanzhu \\
\hline de l'école & 17 & 55 & 29 \\
de l'université & 2 & - & 4 \\
du champ local & 1 & 3 & 5 \\
\hline Professeurs & 6 & 19 & 11 \\
Titulaire & 6 & 1 & 3 \\
Associé & 3 & 2 & 5 \\
Assistant & 1 & 21 & 3 \\
Étudiants & 2 & - & 7 \\
MBA & 2 & 15 & 9 \\
$1^{\text {er cycle }}$ & 20 & 58 & 38 \\
Personnel non académique & Total & res professeurs sont titulaires et il n'y a pas d'étudiants de 1er cycle.
\end{tabular}

Sigles : MBA : Master of Business Administration.

Source : Entretiens réalisés par l'auteur.

Pour être exploités, les entretiens ont été transcrits et découpés en extraits correspondant à une thématique. Pour chacune des écoles, ces extraits ont été groupés et compilés dans un document afin de constituer trois monographies. Différents niveaux de sous-thèmes ont été créés en fonction de la quantité d'extraits contenus dans le corpus d'entretiens. La compilation progressive a révélé l'importance de certains éléments récurrents ou dominants dans les propos des enquêtés. La constitution de ces monographies a ainsi permis de faire apparaître, de manière inductive, les éléments saillants issus du terrain.

Si cet article traite de cas chinois, c'est en raison de l'importance de l'enseignement supérieur sur ce territoire (première population étudiante mondiale), qui demeure proportionnellement encore peu étudié. Mais cela résulte aussi du postulat que, sans oublier l'importance du contexte local, les organisations chinoises peuvent être étudiées comme les autres, en révélant les mécanismes sociaux à l'œuvre, sans avancer d'explications culturalistes ou de " particularité chinoise ». Ainsi, même si l'analyse porte sur des écoles situées en RPC, elles ont été choisies car elles jouissent d'un prestige élevé et s'insèrent dans le champ international des écoles de gestion. Les résultats sont utiles pour comprendre la transformation d'autres écoles prestigieuses, en Europe ou encore en Amérique du Nord ${ }^{5}$. En effet, ces trois cas représentent la diversité des écoles chinoises (trois champs locaux),

5. Ce travail s'inscrit dans le cadre du projet PrestEnce (ANR - 09-SOC-011) mené durant quatre ans (20102013) et visant à étudier la fabrication de la qualité académique dans 27 facultés (ou écoles) en chimie, histoire et gestion, dans cinq pays (France, États-Unis, Italie, et Suisse, ainsi qu'en RPC pour la gestion). 
mais également la variété des positions dans le champ international des écoles de gestion. En comparant la valeur que les unités de gouvernance du champ leur attribue, nous pouvons les distinguer en termes de statut : une école dominante, une importante et une nouvelle venue ( $c f$. Tableau 2 ).

TABleAu 2 - Position des trois écoles dans le champ

\begin{tabular}{lcccc}
\hline & $\begin{array}{c}\text { Nombre } \\
\text { d'années de } \\
\text { classement }\end{array}$ & $\begin{array}{c}\text { Rang } \\
\text { actuel }\end{array}$ & $\begin{array}{c}\text { Date de la lère } \\
\text { accréditation et } \\
\text { nombre d'accréditations }\end{array}$ & $\begin{array}{c}\text { Statut } \\
\text { dans le champ }\end{array}$ \\
\hline ABS & 16 & $+/-20^{\mathrm{e}}$ & $2004(2)$ & Nouveau dominant \\
HKNU Business School & 9 & $+/-30^{\mathrm{e}}$ & $1999(1)$ & Membre important \\
Institut Nanzhu & 0 & $\mathrm{NC}$ & $2010(3)$ & Nouveau venu \\
\hline
\end{tabular}

Référence de classement : Financial Times Global MBA Ranking.

Après avoir montré la construction d'un modèle d'école avec une double orientation académique et professionnelle comme référence à l'échelle internationale (1), nous nous appuierons sur trois études de cas d'écoles chinoises (2) pour montrer comment la division du corps professoral se manifeste comme une réponse à cette prescription du champ international (3).

\section{L'affirmation d'une représentation des écoles à double orientation}

En Amérique du Nord, en Europe, mais aussi en Chine, les écoles de gestion furent le plus souvent le fruit d'initiatives d'élites locales mobilisant leurs ressources (financières, relationnelles) en vue de former des personnes aux compétences nécessaires au développement du tissu économique d'un territoire.

La mission de ces établissements, créés durant la première moitié du $20^{\text {ème }}$ siècle, était l'enseignement professionnel de la gestion par l'apprentissage de la pratique des affaires. En Europe, cet enseignement s'est initialement développé hors des universités. C'est notamment le cas dans des pays comme la Grande Bretagne, l'Allemagne (Locke, 1998), la Suède (Engwall, 1986), une grande partie de la Suisse (Paradeise \& al., 2014), ainsi qu'en France jusqu'au début des années 1960 (Chessel \& Pavis, 2001). Aux États-Unis, les premières écoles de gestion sont nées dans les universités sans pour autant avoir une orientation académique (Khurana, 2007). 


\subsection{Le tournant académique}

À partir de la fin des années 1950, les fondations philanthropiques états-uniennes Ford et Carnegie cherchant à promouvoir la qualité de l'enseignement de la gestion ont milité pour la promotion de nouvelles pratiques des écoles de gestion. Mettant leur forte capacité financière au service de cette mission, ces fondations ont proposé des normes communes synthétisées dans deux rapports ${ }^{6}$ préconisant d'accroître la proportion de professeurs orientés vers la recherche, d'accorder une place plus importante aux analyses quantitatives et aux sciences sociales dans les enseignements, et de développer les formations doctorales pour former les futurs professeurs (Khurana, op. cit., pp .273-282).

Cette orientation académique fut promue aux États-Unis, et dans un contexte de guerre froide, elle fut progressivement exportée dans l'ensemble du monde capitaliste, en Amérique du Nord, en Europe, à Hong Kong, puis en Chine continentale à partir des années 1980 (Soulas, 2017). Alors que leur légitimité tenait à la formation de praticiens de la gestion, les écoles de gestion ont commencé à exploiter leur ancrage académique par leur présence dans les universités pour beaucoup, mais plus généralement par l'orientation vers des activités caractéristiques du monde académique (publications scientifiques, formation doctorale, développement de centres de recherche, etc.).

Cette évolution a progressivement conduit à la coexistence des deux sources de légitimité à l'origine d'une tension croissante entre deux orientations de ces écoles de gestion (Paradeise \& al., op. cit.). Dans les années 1990, ces écoles sont progressivement entrées dans le champ international émergeant à cette époque. Leur comparaison à l'échelle mondiale a accru cette tension, entraînant des changements profonds pour les écoles.

\subsection{Accréditations et classements : les nouveaux acteurs de l'évaluation}

Il existe deux catégories d'unités de gouvernance interne du champ international des écoles de gestion : les agences d'accréditation et les instances de classement.

Une accréditation est " la production d'une déclaration officielle, faisant l'objet d'une publication, concernant la qualité d'une institution ou d'un programme, suite à une évaluation cyclique par des pairs, basée sur des normes reconnues. L'accréditation est censée remplir deux fonctions, le contrôle minimal de la qualité et l'amélioration de l'excellence des institutions " (Cret 2007, p. 26). Les trois accréditations mondialement reconnues pour les écoles de gestion sont : AACSB, EQUIS et AMBA7. Elles ont acquis une importance considérable

6. Les rapports respectivement commandés par les fondations Carnegie et Ford sont : Frank Cook Pierson, The Education of American Businessmen: A Study of University-College Programs in Business Administration, New York, McGraw-Hill, 1959 ; Robert Aaron Gordon \& James Edwin Howell, Higher Education for Business, New York, Columbia University Press, 1959.

7. Le label EQUIS (EFMD Quality Improvement System) est délivré par l'EFMD (voir note n 1). L'accréditation AMBA, à la différence des deux autres, évalue uniquement les MBA et non les écoles dans leur ensemble. 
depuis 1997, date du lancement des premières accréditations EQUIS et de l'exportation de AACSB en Europe (Cret, Ibid., p. 30) et ont étendu leurs activités à la Chine dès la première moitié des années 2000.

Aujourd'hui, le classement d'écoles de gestion le plus influent dans le champ international est celui du Financial Times. Lancé en 1998, il a la double force de s'appuyer sur des données quantitatives et d'avoir un spectre international. Si d'autres classements comme celui de Business Week ont du poids, celui du Financial Times domine clairement le champ international (Kwon \& Easton, 2010). Ces classements sont des outils de mesure acontextuels qui s'appuient sur des métriques telles que le nombre de publications, les salaires de sortie, ou lencore la part d'étudiants étrangers.

En définissant des critères universels pour évaluer les écoles de gestion, ces unités de gouvernance interne instaurent des cadres normatifs au sein du champ, notamment le modèle de la business school, présenté en introduction, auquel ces établissements doivent se conformer pour y acquérir une position plus favorable.

\subsection{La double injonction : des écoles professionnelles et académiques}

En raison de l'enjeu que cette évaluation représente pour les écoles les plus prestigieuses au monde, les critères utilisés par ces agences d'accréditation et instances de classement ont un impact fort sur leurs pratiques. Or, ces organismes veillent à ce que les écoles conservent un équilibre entre leurs orientations respectivement académique et professionnelle.

Comme le stipulent les standards de l'EFMD, « un équilibre adéquat entre la qualité académique et la pertinence managériale est un des principes fondamentaux de EQUIS » (EFMD, 2016, p. 71, notre traduction). Cet équilibre est surtout évalué dans les compétences du corps professoral, qui doivent comprendre les deux orientations. L'accréditation des programmes de MBA par l'Association of MBAs (AMBA) comprend dix principes. Le troisième d'entre eux, "Qualité \& suffisance du corps professoral ", intègre cette double injonction à doter l'école "d'une expertise suffisante et équilibrée en enseignement, recherche et consultance qui guide l'expérience d'apprentissage d'une manière cohésive et intégrée " (AMBA 2016, p. 4, notre traduction).

L'accréditation états-unienne AACSB pousse encore plus loin la logique en théorisant une division des compétences en fonction des deux orientations. Dès 1991, elle a introduit les concepts de "professeur académiquement compétent » et «professeur professionnellement compétent ${ }^{8}$ ". Dans les années 2000, ces désignations ont fait l'objet de trois livres blancs consacrés à l'explicitation des standards sur ce point précis (AACSB, 2006a,b, 2009). Ils définissent une conception des ressources professorales selon une " approche par 'portfolio' ». Pour construire ce " portfolio de contributions intellectuelles » au sein

8. Concepts "d'academically qualified faculty " et "professionally qualified faculty ", abrégés en "AQ et PG faculty ». Détaillés dans le stardard n 10 (AACSB, 2003, pp.42-51). 
du corps professoral dans son ensemble", une école doit maintenir un équilibre entre les deux orientations dans les compétences de $90 \%$ du corps professoral où au moins $50 \%$ des professeurs ont une orientation académique (AACSB, 2003, p. 42).

Dans les standards adoptés depuis 2013, AACSB a encore affiné sa théorisation en créant quatre profils de compétences professorales. Elle distingue toujours les « académiques » et les « praticiens » en déclinant chacun en fonction de la nature de leur formation et de leur type de mission au sein de l'école. Les " académiques " sont les titulaires d'un doctorat qui, selon leur activité principale (académique ou professionnelle), sont désignés comme Scholarly Academics (SA) ou Practice Academics (PA). Les praticiens, quant à eux, sont ceux qui n'ont pas de doctorat, mais une expérience professionnelle significative. Selon qu'ils ont une activité plutôt de recherche ou d'enseignement, ils sont respectivement désignés comme Scholarly Practicioners (SP) ou Instructional Practicioners (IP). Les écoles sollicitant l'accréditation doivent classer leurs professeurs dans ces catégories en respectant certains équilibres ${ }^{10}$.

Le classement du Financial Times, quant à lui, pousse les écoles à exceller dans les deux orientations. Il privilégie notamment deux critères : l'alumni carrer progress, fondé sur la satisfaction et l'insertion des étudiants sur le marché du travail et qui relève largement de l'orientation professionnelle, et l'idea génération, associé à l'orientation académique. Pour préserver ou accroître leur rang, les écoles doivent réussir à marquer plus de points que les autres dans ces deux catégories.

\section{Trois écoles de gestion chinoises dans le champ international}

La position dans la hiérarchie du champ international des écoles de gestion est un élément qui transforme le rapport des écoles aux préconisations de ses unités de gouvernance interne. Pour comprendre la transformation du corps professoral des écoles chinoises, nous allons nous appuyer sur les cas de trois établissements ayant des positions différentes dans le champ international. En RPC, ces écoles évoluent dans plusieurs champs d'action stratégique locaux encadrant ces établissements. Il existe ainsi celles qui se trouvent dans le système universitaire de Chine continentale, celles du système universitaire de Hong Kong et celles qui ne sont pas rattachées à une université. Les trois études de cas portent sur une école de chaque catégorie.

9. Ces expressions se retrouvent dans les trois livres blancs mentionnés depuis 2006 ainsi que dans les standards de AACSB adopté en 2003 et 2013.

10. $90 \%$ des professeurs doivent correspondre à une de ces quatre définitions avec un minimum de $40 \%$ de SA, et un maximum de $60 \%$ de IP (standard n 15, AACSB 2013, p. 45). 


\subsection{Dans le système universitaire de Chine continentale}

En Chine continentale, les grandes universités héritières du système maoïste ont commencé à ouvrir des facultés de gestion à partir des années 1980. Dans ce système universitaire public qui comprend plusieurs milliers d'établissements, les universités sont étroitement régulées par le gouvernement national ou provincial qui assure l'essentiel de leur financement. Le ministère de l'Education est ainsi l'unité de gouvernance interne de ce champ local. Parmi ces facultés, plus d'une centaine dispense une formation de premier cycle en gestion et au moins un MBA.

Situé à Canton, l'Institut Nanzhu est une faculté de gestion de la plus prestigieuse université du Sud de la Chine. Elle a été créée en 1989, grâce au soutien de Cantonais de la diaspora états-unienne réunis au sein d'une fondation philanthropique. Elle compte plus de 3000 étudiants et une centaine de professeurs, tous Chinois du continent majoritairement formés dans les universités locales. L'école offre des programmes universitaires de la licence au doctorat, ainsi qu'une large gamme de MBA, d'Executive $M B A(\mathrm{EMBA})^{11}$ et de formations courtes pour les entreprises dont les frais sont très élevés. L'école ne figure pas encore dans le classement mondial du Financial Times, malgré ses efforts en ce sens, mais son MBA est classé dans les dix premiers de Chine continentale par le magazine Forbes. Depuis 2010, son accréditation par les trois agences reconnues dans le champ international en fait un nouveau venu dans le champ.

\subsection{Dans le système universitaire de Hong Kong}

Il existe aujourd'hui deux systèmes universitaires en Chine. Hong Kong fait partie de la RPC depuis sa rétrocession en 1997. Cependant, suivant le principe d' " un pays, deux systèmes ", le gouvernement local conserve l'autonomie de sa politique universitaire. Au sein de ce système, l'enseignement de la gestion s'est développé, à partir des années 1960, avec la création d'une première faculté de gestion, puis une demi-douzaine d'autres dans les universités publiques locales à partir des années 1990. Ces établissements sont essentiellement financés par les dotations publiques du gouvernement par l'intermédiaire d'une agence (University Grants Committee ${ }^{12}$ ) qui évalue les universités et répartit les fonds publics. Cette agence assure la gouvernance de ce champ local des écoles de gestion universitaires de Hong Kong.

HKNU Business School est une faculté d'une grande université publique : Hong Kong National University. Elle compte plus de 4000 étudiants et une centaine de

11. Les MBA sont des programmes d'un an ou deux à destination de jeunes actifs (d'environ 30 ans), alors que les EMBA sont destinés à des cadres plus âgés et ayant des postes à plus haute responsabilité.

12. Commission de financement des universités. 
professeurs permanents, à $80 \%$ d'origine chinoise (Hong Kong, continent et outremer). Ils sont pour la plupart diplômés en Occident ou à Hong Kong.

Comme les autres facultés, l'école propose des programmes universitaires (licence, master, doctorat) dont les frais sont fixés par l'État. Elle dispense également des programmes professionnels comme son MBA ouvert depuis 1966, mais aussi des EMBA et des formations courtes pour les entreprises.

L'école est accréditée par l'AACSB depuis près de 20 ans et est régulièrement classée par le Financial Times parmi les 30 premières mondiales. Établie comme une des écoles qui comptent dans le champ international, HKNU Business school en est un membre important.

\subsection{Parmi les écoles indépendantes}

En RPC, une dizaine d'écoles ont été créées en dehors des systèmes éducatifs. C'est le cas de Asia Business School (ABS), ouverte à Shanghai en 1994, qui n'est pas une école universitaire. Héritière d'un centre de formation sino-européen, c'est aujourd'hui une école de gestion indépendante du ministère de l'Éducation. Son activité est régulée par la législation sur les activités commerciales, comme c'est le cas de quelques autres écoles sur le continent. Elle est co-dirigée par une association d'écoles européennes et le gouvernement chinois. Dans le champ local de ces " $M B A$ schools», la gouvernance fonctionne, dans un contexte régulé par le ministère du Commerce, largement sur l'offre et la demande de formation parmi les jeunes actifs chinois.

En raison de ce statut, Asia Business School n'est pas autorisée à délivrer des programmes universitaires régulés par le ministère de l'Éducation (licence, master et doctorat). Elle compte néanmoins près de 1500 étudiants dans plusieurs programmes professionnels (MBA, EMBA, et formations aux entreprises) dont plus de la moitié en EMBA. La centaine de personnels académiques de l'école est aux trois quarts composée de professeurs seniors en fin de carrière, la moitié étant permanents. Tous diplômés en Occident (majoritairement aux États-Unis), un peu plus de la moitié est d'origine chinoise. L'école s'est fait connaître récemment en figurant régulièrement parmi les vingt premières écoles mondiales du Financial Times Global MBA ranking. Elle a également obtenu les labels des deux plus importantes agences d'accréditation du champ international (EQUIS et AACSB), ce qui la place depuis peu parmi les membres dominants du champ international. 


\section{Vers une scission du corps professoral dans les écoles chinoises}

Chacune de ces trois écoles se trouve dans un champ local qui l'oriente plutôt vers les activités professionnelles (école indépendante de formation pour adultes comme ABS) ou académiques (facultés universitaires comme HKNU Business School et l'Institut Nanzhu). Néanmoins, leur intégration dans le champ international les oblige à répondre aux deux injonctions, principalement à travers le recrutement de professeurs.

Cependant, un professeur qui publie régulièrement des articles dans des revues de rang "A ", tout en ayant une connaissance du monde des affaires lui permettant de dispenser avec succès des cours de MBA et EMBA, correspond désormais au profil idéal, mais n’est pas un objectif de recrutement.

Pour ne pas perdre la possibilité d'être bien évaluées par les unités de gouvernance du champ international, les écoles répartissent ces compétences à l'échelle de l'organisation. Il s'agit de recruter des professeurs qui excellent dans l'une des deux orientations, et d'avoir ainsi les deux compétences à un très haut niveau réunies au sein de l'école. Cette approche correspond à ce que préconise l'agence AACSB qui accrédite les trois écoles étudiées. Cette logique, enjointe par la gouvernance du champ international, transforme alors l'organisation des écoles. Ces dernières mettent en place une division de leur corps professoral se traduisant par la création de deux parcours de carrière sur la base de deux missions : recherche et professionnelle. Néanmoins, l'intégration de cette règle organisationnelle varie selon la position des écoles dans le champ international.

\subsection{Dans les universités continentales : de l'ancien au nouveau système}

Comme nous l'avons expliqué en introduction, l'Institut Nanzhu est une faculté universitaire de Chine continentale, encore peu élevée dans la hiérarchie du champ international. Linfluence du champ local (i.e. le système universitaire continental) sur le recrutement de ses professeurs y est plus fort que dans les deux autres établissements.

Dans les universités continentales, les professeurs ont un emploi stable avec un salaire fixe très faible et ils complètent leur revenu par une rémunération à la pièce de leurs activités. Chaque cours enseigné, chaque article publié leur rapporte de l'argent supplémentaire. Ces allocations varient en fonction de la difficulté du cours (premier cycle ou EMBA) ou du prestige de la publication (en fonction d'un classement des supports de publication). Les jeunes professeurs se concentrent généralement sur les activités de recherche pour obtenir une promotion, alors qu'une fois professeur associé ou titulaire, ils ont plus de possibilités pour compléter leurs revenus par des activités professionnelles en entreprise et enseigner dans les programmes professionnels (Soulas, 2016, pp. 211-216). Ce système, hérité de la période maoïste, permet de préserver l'orientation professionnelle des 
professeurs de gestion, mais conduit certains d'entre eux à déconsidérer les exigences de recherche croissantes en complétant leur revenu par des activités professionnelles externes.

Dans la plupart des universités prestigieuses, cette situation est remise en cause, sous l'impulsion du ministère de l'Éducation, unité de gouvernance interne de ce champ local, qui souhaite que les universités investissent plus dans les activités de recherche. Lancés en 2003 à l'Université de Pékin sous le nom de " réforme de Beida ${ }^{13}$ ", de nouveaux systèmes de carrière sont mis en place dans la plupart des grandes universités chinoises. Ils se distinguent de l'ancien système sur deux dimensions majeures : la stabilité de l'emploi n'est plus formellement garantie et la rémunération devient fixe et plus élevée (Merle, 2004). Les facultés adaptent ces règles, entraînant une coexistence des anciens et nouveaux systèmes en leur sein. Les écoles de gestion sont généralement les plus avancées dans ces réformes car elles mobilisent leurs ressources propres largement issues des programmes professionnels pour financer le nouveau système. Les nouveaux salaires, plus proches des standards internationaux, ont également pour objectif de recruter au-delà des frontières de la Chine continentale et de répondre au désir d'internationalisation des écoles de gestion chinoises. Un nouveau système de carrière parallèle s'affirme progressivement, visant à favoriser l'orientation recherche des professeurs, freinée par le système hérité de l'administration communiste.

L'Institut Nanzhu a introduit son nouveau système de salaire à la fin des années 2000. Il a pour objectif de recruter des " tortues de mers ${ }^{14}$ ", terme désignant les Chinois diplômés à l'étranger. Il a, dans un premier temps, constitué un second mode de recrutement où certains profils étaient recrutés avec un système de salaire " local » et d'autres avec un système " international ». En 2013, le doyen a mis fin aux recrutements "locaux ", systématisant ceux sur des salaires fixes qui sont désormais la norme. Ce système est autofinancé à l'échelle de la faculté ${ }^{15}$, mais encouragé par l'université. Il est également proposé sur une base volontaire aux professeurs déjà en poste, probablement pour éviter qu'il se traduise par une division entre tortues de mers (海龟, haigui) et Chinois formés en Chine. "Les professeurs chinois seraient très contrariés si nous offrions un salaire très élevé aux professeurs extérieurs [nouvellement recrutés] ${ }^{16} "$.

Comme dans d'autres universités, si le doyen considère que le nouveau système fonctionne, l'ancien va probablement disparaître lorsque ses dernières recrues partiront à la retraite. Cependant, dans l'ancien système, les professeurs seniors pouvaient compléter

13. 北大改革, beida gaige. Le diminutif désignant l'Université de Pékin (北京大学, beijing daxue) est beida (北大).

14. Les Chinois revenus de l'étranger sont désignés par le terme de 海龟 (haigui) qui signifie " tortues de mer ", jeu de mots avec le terme homophone 海归 (haigui) qui signifie "revenant par la mer » : le son gui peut signifier à la fois « revenir » (归) et « tortue " (龟).

15. La faculté ne se substitue pas au salaire fixe versé par l'État, même si elle verse un salaire complémentaire important pour atteindre un salaire total élevé.

16. Entretien avec Li Meihong, Directeur adjoint MBA Center, Institut Nanzhu. 
leur salaire par des cours professionnels et des activités dans le monde des affaires. La suppression de ces compléments de salaire fait de la recherche l'activité prioritaire de ces professeurs. Les jeunes ayant moins d'opportunités pour compléter leur salaire par l'orientation professionnelle, ils sont majoritairement favorables à ce nouveau système :

"Avec ce salaire, si vous avez des cours ou des papiers [publiés], vous n'avez pas plus d'argent. Parce que tout est inclus. Donc ça, peut-être que pour les jeunes professeurs, ils pensent que c'est une bonne opportunité, parce que chaque année, il y a un salaire fixe et vous n'avez pas à dédier tout votre temps aux cours. Parce que sans cette situation, vous devez avoir plus de cours pour gagner de l'argent. Mais dans cette situation, vous pouvez consacrer la plupart du temps à écrire des papiers. Donc, vous n'avez pas besoin d'avoir plus de cours pour gagner de l'argent. " (Entretien avec Lu Guotao, professeur associé, Institut Nanzhu)

Contrairement à ce qui se produit à $\mathrm{ABS}$ et HKNU Business School, la division du corps professoral ne va pas faire émerger un deuxième parcours, mais vise à se substituer progressivement à l'existant. Les unités de gouvernance du champ international n'ont pas encore une influence très forte, mais cette situation devrait évoluer par l'ascension de l'école dans ce champ, mais aussi par la création d'une accréditation gouvernementale des MBA « utilisant les standards AACSB et EQUIS comme étalon " (Chen \& Yang, 2010, p. 135 , notre traduction).

\section{2 À Hong Kong : I'internalisation d'un parcours professionnel}

Dans le système universitaire de Hong Kong, le Bureau de l'Éducation, qui gouverne le champ local, oriente également les universités vers la recherche. Traditionnellement, les professeurs des universités hongkongaises sont recrutés dans une logique de carrière évaluée sur ce critère. Ce parcours recherche (aussi appelé research track) débute au poste de professeur assistant, avec des possibilités de promotion en tant qu'associé, puis professeur titulaire (full professor). Ces professeurs ont une mission principale de recherche : leur promotion dépend essentiellement de cette activité. À HKNU Business School, les exigences dans ce domaine sont si fortes que l'école a recruté un grand nombre de non-permanents pour assurer la pertinence professionnelle de la formation.

Les instructeurs sont employés sur des contrats d'enseignement à durée déterminée, avec une charge d'enseignement nettement plus élevée que les professeurs. Ce sont des contrats précaires qui existent dans toute l'université. Pour l'école, ils permettent d'externaliser la mission d'enseignement en premier cycle à faible coût et de décharger les professeurs permanents qui se concentrent ainsi sur la publication d'articles.

Un second statut d'enseignant non permanent est spécifiquement dédié aux cours de MBA et EMBA : les affiliés (ou adjuncts). Ces personnels sont liés à l'école par un contrat à temps partiel incluant des personnes dont l'activité d'enseignement est régulière et d'autres qui interviennent de manière ponctuelle. Ce type de statut destiné à des professionnels du monde des affaires existe dans nombre d'écoles de gestion dans le monde, 
notamment en France et aux États-Unis (Delemarle \& Paradeise, 2011 ; Mignot-Gérard \& Cauchard, 2014), mais est très peu utilisé dans le reste de l'université car étroitement associé à l'orientation professionnelle. Au sein de l'école de gestion, leur rôle est double : ils complètent l'effectif enseignant et assurent une connexion avec le monde des affaires.

Depuis le milieu des années 2000, HKNU Business School recrute de plus en plus de personnels non permanents (essentiellement des affiliés) qui représentent désormais la moitié des effectifs totaux. Lécole ne peut pas externaliser sa mission d'enseignement au-delà d'un certain niveau (notamment en raison des exigences de l'accréditation AACSB). Or, la position de l'école dans le champ international s'est trouvée menacée depuis le milieu des années 2000, alertant le doyen sur les carences de compétences d'enseignement dans les MBA et EMBA qui assurent une part importante des revenus à la faculté de gestion. Cependant, le recrutement des professeurs de la tenure track passe en premier lieu par les départements ${ }^{17}$ dont la dotation est fondée sur la productivité des professeurs en termes de recherche. Pour préserver ses revenus issus des programmes professionnels et rééquilibrer les recrutements, le doyen de l'école a créé un mode de recrutement supplémentaire.

"Quelquefois, la faculté aimerait que les départements recrutent des profils différents en disant "Nous on a besoin de gens avec un profil capable d'enseigner dans les programmes de MBA" et les départements vont dire "Oui, mais nous on a besoin de gens qui sont capables de faire de la recherche dans tel ou tel domaine" et parfois ça ne correspond pas, donc ça crée un peu des, je dirais des lignes de tension entre la faculté et les départements. "

Et comment ça se traduit ces lignes de tensions?

"Ça se traduit par... par la faculté qui engage des gens pour enseigner les cours en dehors du département. " (Entretien Marc SANIA, professional consultant, HKNU Business School)

La faculté de gestion a mobilisé ses ressources propres pour mettre en place un parcours professionnel en recrutant des personnels, indépendamment du parcours recherche géré par les départements. Sous le nom de professional consultant, ces profils ont une formation académique, mais sont recrutés pour leur orientation professionnelle. Ce titre, unique au sein de l'université, est également une originalité illisible dans le champ international : "Le problème avec professional consultant, c’est que personne ne sait ce que ça veut dire $^{18} »$. Cette situation a conduit l'école à s'aligner sur les pratiques d'autres écoles de gestion, notamment états-uniennes, qui ont créé des postes de professor of practice, dont la signification est proche. Le nombre a augmenté pour passer à neuf et une hiérarchie

17. La première étape du recrutement est gérée par les départements, les autres acteurs (faculté, puis université) interviennent après pour confirmer ou invalider cette sélection. Contrairement à la désignation française, en RPC comme dans la plupart des pays anglo-saxons, les départements sont des unités chargées de la formation et de la recherche.

18. Entretien avec Marc Sania, professional consultant, HKNU Business School. 
interne a été mise en place à partir de 2013 entre associate professor of practice et professor of practice.

À la différence des affiliés ou des instructeurs, ces personnels sont permanents et officiellement intégrés dans le corps professoral. Ils sont payés et évalués par l'école (et non le département) avec une mission principale liée à l'orientation professionnelle (enseignements et administration de programmes professionnels notamment), sans exigence en termes de recherche. Cette division des activités crée une fracture au sein du corps professoral. Ces professeurs ne partagent pas la vie du département, centrée sur la recherche scientifique : "Il n'y a pratiquement plus d'échanges entre moi et les autres collègues du département de management parce que, institutionnellement on n'est plus.. un groupe. ${ }^{19}$. Pour de nombreux professeurs, cette fracture est considérée comme un mal nécessaire pour se positionner favorablement dans le champ international au regard des évaluations de l'orientation respectivement professionnelle et académique.

\subsection{L'émergence d'un parcours recherche dans l'école indépendante}

À l'origine, Asia Business School était un centre de formation professionnel fonctionnant sur la base de professeurs temporaires. Son MBA, classé parmi les meilleurs d'Asie, l'a fait entrer très rapidement dans le champ international, malgré une faible orientation académique. École autofinancée, la réussite de ses programmes professionnels garantit sa stabilité financière.

Durant les premières années, le corps professoral fonctionnait sur un "modèle volant» : les professeurs venaient en avion depuis l'Europe, dispensaient leurs cours et repartaient sans vivre sur place. Néanmoins, cette situation limitait l'identification de l'école comme un acteur de la recherche car peu de professeurs menaient des activités de recherche au nom d'ABS.

Ses responsables ont donc cherché à limiter le recours à ce modèle qui, aujourd'hui, ne concerne plus la majorité des professeurs. Depuis la fin des années 2000, ils développent un nouveau mode de recrutement avec une orientation recherche et de véritables postes permanents. Ce tournant a débuté en 2005, avec la création de départements disciplinaires analogues aux universités. Alors que les programmes (MBA, EMBA, ...) ont toujours eu un rôle structurant, les départements commencent à prendre de l'importance dans l'organisation. Fonctionnant initialement comme des coquilles vides, ils sont désormais acteurs du processus de recrutement des professeurs assistants et associés.

En parallèle, les doyens successifs essayent de structurer un parcours professionnel en créant des statuts de professeurs permanents fonctionnant sur un autre mode d'évaluation. Alors que ces profils n'étaient pas définis auparavant, ABS s'est mis au dia-

19. Ibid. 
pason des écoles dominantes du champ international en créant des postes de professor of practice. Lidée est de mettre ces personnes au même niveau que les professeurs du parcours recherche, mais avec une orientation professionnelle. Les membres de l'école ont intégré cette nouvelle catégorie des professors of practice car elle émane directement de la gouvernance du champ international. "Je pense qu'il y a désormais de la pression de l'AACSB pour mettre en avant ce que nous appelons 'professor of management practice" 20 ". Ces professeurs sont évalués par le doyen (et non le département) sur leur capacité à apporter la pertinence professionnelle au sein de l'école. En ce sens, ils complètent la gamme de compétences ou le "portfolio de contributions intellectuelles ", selon les termes de AACSB que les dirigeants de l'école veulent construire. C'est la conviction du doyen d'ABS :

"Donc, je vois plus cela comme un continuum, où vous avez des gens qui sont très orientés recherche et vous avez des gens très praticiens, n'est-ce pas? Et pour moi, mais c'est l'opinion de Friedrich Brandt, [il sourit] une bonne école a un large panel de personnes, d'un extrême à l'autre [il balaye l'espace entre ses deux doigts représentant les extrêmes] parce que nous voulons tous avoir des supers chercheurs qui sont aussi de supers enseignants. Vous en avez, mais vous savez, très très [insiste sur ces deux mots] rarement. Et c'est dans ce sens que la communauté des écoles de gestion est allée. " (Entretien Friedrich Brandt, doyen, ABS)

ABS, qui domine dans le champ international, cherche un nouvel équilibre entre les orientations respectivement professionnelle et recherche. Pour cela, ses dirigeants formalisent des recrutements dans un parcours recherche inspiré des pratiques universitaires. Ils s'appuient sur les unités de gouvernance interne et les titulaires du champ international pour mettre en place un parcours professionnel lisible à cette échelle.

\section{Conclusion}

Cette étude concerne des écoles très différentes les unes de autres (indépendantes ou universitaires, très prestigieuses ou de rayonnement régional), mais qui revendiquent une place dans le champ international des écoles de gestion. Pour cela, elles doivent suivre le modèle de la business school, défini par les unités de gouvernance interne de ce champ que sont les agences d'accréditation et instances de classement. Elles doivent non seulement s'assurer que leur corps professoral a une orientation académique et professionnelle, mais aussi qu'il excelle dans les deux domaines.

Les établissements ayant acquis une place privilégiée dans le champ international suivent de près ces préconisations, alors que les nouveaux venus sont encore très dépendants des règles de leur champ local qui reste le principal garant de leur légitimité. Ainsi, à l'Institut Nanzhu, qui entre à peine dans les règles du jeu international, la division du corps professoral est largement dictée par les réformes du système univer-

20. Entretien avec Sonny Bradford, professor of practice, ABS. 
sitaire de Chine continentale. À l'image des autres écoles de ce champ local, Nanzhu développe son orientation recherche sans compenser par un recrutement de profils à orientation professionnelle.

HKNU Business School est depuis longtemps un acteur du champ international plus à l'écoute des évaluations de ses unités de gouvernance. Sa forte orientation recherche, qui caractérise l'ensemble des écoles du système universitaire hongkongais, a créé un déséquilibre qui se manifeste à travers la perte temporaire de reconnaissance de son MBA. Lécole a rééquilibré la situation en s'inspirant des pratiques de recrutement des professors of practice du champ international, d'abord sous un autre nom, puis en reprenant les termes inspirés par l'AACSB.

Asia Business School (ABS) est dans un champ d'écoles indépendantes très orienté vers le monde professionnel où les activités de recherche sont optionnelles. Première école d'Asie dans la hiérarchie du champ international, elle suit à la lettre les préconisations du modèle de la business school. Non seulement elle investit dans les activités de recherche, mais elle reprend à son compte la théorisation des unités de gouvernance interne impliquant une division organisée du corps professoral sur la base des deux orientations.

Abandonnant la quête du professeur "super-star ", la solution proposée aux écoles pour suivre le modèle de la business school est donc simple : des professeurs professionnels et des professeurs académiques, le tout dans une seule organisation. Cette scission du corps professoral se décline de façon variable selon les établissements, en fonction de leur place dans le champ international. Elle chamboule les équilibres organisationnels en créant des cloisonnements et soulève les premières inquiétudes sur les effets secondaires de ces mesures.

Cet article s'appuie sur des cas chinois, mais les résultats du projet PrestEnce ( $c f$. note 4) montrent que ces transformations se retrouvent dans les écoles d'Europe et d'Amérique du Nord les plus prestigieuses (Mignot-Gérard \& Cauchard, op. cit.). Ainsi, dans une école française parmi les plus importantes du champ international, un ancien haut responsable décrit une scission similaire avec l'exemple de professeurs non chercheurs, recrutés pour assurer l'orientation professionnelle :

"Quelqu'un qui ne rentre pas dans le jeu de la chasse à la tenure ${ }^{21}$, qui a un contrat pour enseigner, est membre du corps professoral, mais pas autant que les gens qui vont faire la tenure. [...] Il apporte un service inestimable : il contribue à donner une réponse à ce problème mécanique. [...] Voilà donc une réponse organisationnelle émergente qui rend plus facile la mise en auvre de la stratégie du marché. " (Extrait d'entretien, Delemarle \& Paradeise, op. cit., p. 155)

21. i.e. Titularisation. 
Pour toutes les écoles de gestion du monde, les unités de gouvernance interne du champ international définissent un modèle de "bonne " école, en supposant qu'un et un font deux. Autrement dit, deux compétences additionnées au sein d'une organisation seraient équivalentes à celles de professeurs qui seraient à la fois chercheurs en gestion et connectés au monde de l'entreprise. Cette équation représente une approche très discutable pour les missions d'enseignement et de recherche des écoles, mais s'impose comme le chemin à emprunter pour toutes les écoles souhaitant s'affirmer dans le monde des business schools.

\section{Bibliographie}

AACSB (2003), Eligibility Procedures and Accreditation Standards for Business Accreditation (2012 update), p. 78.

AACSB (2006a), Deploying Academically Qualified Facult : An Interpretation of AACSB Standards, p. 24.

AACSB (2006b), Deploying Professionally Qualified Faculty : An Interpretation of AACSB Standards, p. 15.

AACSB (2009), AQ/PQ Status: Establishing Criteria for Attainment and Maintenance of Faculty Qualifications. An Interpretation of AACSB Accreditation Standards, p. 21.

AACSB (2013), Eligibility Procedures and Accreditation Standards for Business Accreditation (2016 update), p. 53.

AMBA (2016), MBA Accreditation Criteria, Association of MBAs, p. 14.

Blanchard M. (2012), Socio-histoire d'une entreprise éducative : le développement des Ecoles supérieures de commerce en France (fin du XIXe siècle-2010). Thèse de doctorat en sociologie, EHESS.

Chen X. \& Yang B. (2010), “Copying from others or developing locally?: Successes and challenges of MBA education in China (1990-2010)", in Journal of Chinese Human Resources Management 1.2, pp. 28-145.

Chessel M.-E. \& Pavis F. (2001), Le Technocrate, le patron et le professeur : Une histoire de l'enseignement supérieur de gestion, Paris, Belin.

Cret, B. (2007), L'émergence des accréditations : origine et efficacité d'un label. Thèse de doctorat, Paris, IEP Paris.

Delemarle, A. \& Paradeise C. (2011, Monographie de l'École, Gestion, France, 2010. Monographie réalisée pour le projet ANR PrestEnce, p. 259.

EFMD (2016), EQUIS Standards \& Criteria 2016. EFMD, p. 76. 
Engwall L. (1986), "Mercury meets Minerva", in Scandinavian Journal of Management Studies 3.2, pp. 121-138.

Fligstein, N. \& McAdam D. (2012), A Theory of Fields, New York : Oxford University Press.

Khurana R. (2007), From higher aims to hired hands: The social transformation of american business schools and the unfulfilled promise of management as a profession. Princeton University Press.

Kwon W. \& Easton G. (2010), "Conceptualizing the role of evaluation systems in markets: The case of dominant evaluator", in Marketing Theory 10.2, pp. 123-143.

Locke, R. R. (1998). Management Education. Dartmouth Publishing Company.

Merle A. (2004), « La réforme des Universités et de l'enseignement supérieur en Chine : le débat autour de l'Université de Pékin ", Lettre du CEFC, Nouvelles de Chine, MarsAvril, p. 6.

Mignot-Gérard S. \& Cauchard L. (2014), “The race for research productivity and the segmentation of the academic profession in three top business schools (France, Italy and the U.S.)", in 30th EGOS colloquium, Rotterdam School of Management, Netherlands.

Paradeise C. \& al. (2014), "Relevance and excellence in higher education vocational schools : Business schools as institutional actors", in The institutional development of business schools. Sous la dir. de Pettigrew A. M., Cornuel E. \& Homme U, London: Oxford University Press.

Soulas T. 2016), "Business schools Made in China", L'émergence des écoles de gestion chinoises, thèse de doctorat en sociologie, Université Paris Est.

Soulas T. (2017), "Une leçon de soft power : le rôle des coopérations états-uniennes avec la Chine dans l'exportation de l'enseignement de la gestion ", in Revue Internationale des études du développement, 231, pp. 125-147. 